\title{
СРАВНЕНИЕ РЕЗУЛЬТАТОВ РАННЕЙ И ОТЛОЖЕННОЙ ЛАПАРОСКОПИЧЕСКОЙ ХОЛЕЦИСТЭКТОМИИ ДЛЯ ЛЕЧЕНИЯ ОСТРОГО ХОЛЕЦИСТИТА: МЕТА-АНАЛИЗ ПРОСПЕКТИВНЫХ РАНДОМИЗИРОВАННЫХ КОНТРОЛИРУЕМЫХ ИССЛЕДОВАНИЙ
}

\author{
Манзюк А.В.
}

Состояние вопроса: в предыдущих исследованиях, посвященных теме сроков проведения холецистэктомии при остром холецистите, проводился анализ лишь части существующих рандомизированных контролируемых исследований и не анализировались различия в экономическом эффекте. Целью данной работы было определить, какие сроки оперативного вмешательства являются оптимальными при лечении острого холецистита.

Материаль и методы: в базах данных PubMed, EMBASE, Cochrane Library u Web of Science проводился поиск рандомизированных контролируемых исследований, в которых проводилось сравнение результатов лечения острого холецистита при ранней и отложенной лапароскопической холечистэктомии.

Результаты: было найдено 1356 статей, из которых для мета-анализа было отобрано 17 проспективных рандомизированных контролируемых исследований, в которых наблюдались 1756 пачиентов. Наблюдалось снижение среднего количества койко-дней (Разность средних: -3, 10; 95\% ДИ-3,51,2,69) и затрат на лечение (Стандартизованная разность средних: -0,78; $95 \%$ ДИ -1,31; -0,26) для паџиентов из группь ранней лапароскопической холецистэктомии. Различий в смертности, общем количестве осложнений, переводов в лапаротомию, ятрогенном повреждении желчных протоков и средней длительности оперативного вмешательства не наблюдалось.

Заключение: применение ранней лапароскопической холецистэктомии для лечения острого холецистита (в течение первой недели после появления симптомов) значительно сокращает количество времени и средств, затраченных на лечение пациента, не создавая повышенного риска возникновения любых интра- и постоперационных осложнений. 
Ключевые слова: острый холецистит; лапароскопическая холеизистэктомия; мета-анализ.

\section{COMPARISON OF RESULTS OF EARLY AND DELAYED LAPAROSCOPIC CHOLECYSTECTOMY FOR TREATMENT OF ACUTE CHOLECYSTITIS: META-ANALYSIS OF PROSPECTIVE RANDOMIZED CONTROLLED TRIALS}

\section{Manziuk A. V.}

Background: in the previous researches on subject of terms of cholecystectomy in acute cholecystitis only part of all existing randomized controlled trials were analyzed, economic effect was not examined. The objective of this study was to find optimal terms of operative intervention in acute cholecystitis.

Materials and methods: databases PubMed, EMBASE, Cochrane Library $u$ Web of Science were searched for randomized controlled trials, which compared results of treatment of acute cholecystitis with early and delayed laparoscopic cholecystectomy.

Results: 17 prospective randomized controlled trials with 1756 patients were included in meta-analysis. Decrease in mean hospital stay (Mean difference $-3,10 ; 95 \%$ CI -3,51,-2,69) and cost of treatment (Standardized mean difference -0,78; $95 \%$ CI -1,31; -0,26) were found for patients from group of early laparoscopic cholecystectomy. Differences in mortality, overall complications, conversion rate, iatrogenic bile injury and mean operative time were not found.

Conclusion: applying of early laparoscopic cholecystectomy in treatment of acute cholecystitis (during the first week after appearing of symptoms) noticeably decrease amount of time and money, spent on treatment of patient, not creating an increased risk of complications.

Keywords: acute cholecystitis; laparoscopic cholecystectomy; meta-analysis.

\section{Введение}

Желчнокаменная болезнь широко распространена во всем мире, причем наибольшая распространенность наблюдается в США (около $15 \%$ населения) [1], в странах Европы (5,9-21,9\% населения) [2] и в России (оценивается в 12\% населения) [3]. Острый холецистит - заболевание, которое в $90 \%$ случаев является осложнением желчнокаменной болезни 
[4]. Оптимальной тактикой лечения острого холецистита является оперативное вмешательство. В России ежегодно проводится от 350 до 500 тысяч холецистэктомий, при этом смертность составляет не более $1,5 \%$ благодаря внедрению малоинвазивных оперативных техник [5].

Оптимальным методом оперативного лечения острого холецистита является лапароскопическая холецистэктомия. Однако сроки, в которые должна быть выполнена операция, остаются спорными [6], так как результаты многих исследований говорят о большем количестве интра- и постоперационных осложнений, большей частоты переводов операции из лапароскопической в лапаротомическую, об удлинении времени операций и большей сложности оперативного вмешательства для хирурга при раннем проведении операции по сравнению с операциями, отложенными на срок от 6 до 12 недель для проведения консервативного лечения [6, 7-10].

\section{Цель работы}

Целью этого мета-анализа было выявление различий между ранней и отложенной лапароскопической холецистэктомией в летальности, общем количестве осложнений, частоте ятрогенного повреждения желчных протоков, а также в общем количестве дней, проведенных в больнице (далее койко-дней), общем времени операции, переводах операции из лапароскопической в лапаротомическую и в общих финансовых затратах на проведение оперативного вмешательства на основе данных проспективных рандомизированных контролируемых исследований (РКИ).

В предыдущих исследованиях на иностранном языке $[11,12]$ уже проводилось сравнение результатов лечения в группах ранней и отложенной лапароскопической холецистэктомии, однако ни в одном из них не исследовались различия в экономическом эффекте. Кроме того, полный объем существующих проспективных РКИ, сравнивающих результаты лечения при ранней и отложенной лапароскопической холецистэктомии не исследовался ранее ни в одной из существующих работ, необходимый размер выборки по большинству характеристик также не был достигнут ранее.

\section{Материалы и методы}
А) Источник данных и методика поиска.

Поиск проводился по базам данных PubMed, EMBASE, Cochrane Library, в том числе Cochrane Central Register of Controlled Trials (CENTRAL) и Web of Science с использованием соответствующих теме исследования терминов на английском языке: "acute cholecystitis", "laparoscopic 
cholecystectomy", "early" и "delayed”. Отбирались проспективные рандомизированные контролируемые исследования, сравнивающие результаты лечения при ранней и отложенной лапараскопической холецистэктомией и опубликованные до 1 августа 2018 года. В ходе поиска по базам данных были также вручную просмотрены похожие статьи, а также статьи, упоминаемые в релевантных исследованиях. Были найдены 1356 статей, из которых в мета-анализ были включены 17.

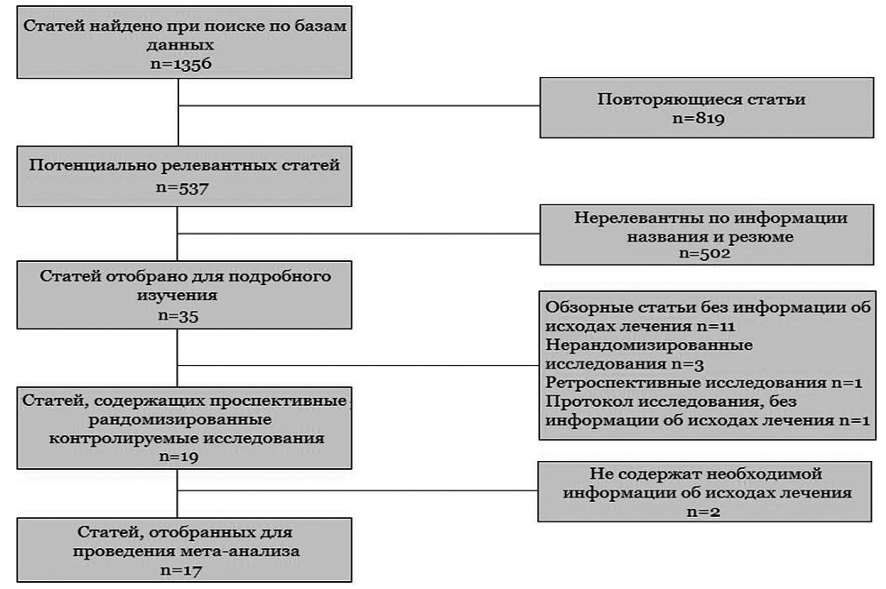

Pис. 1. Блок-схема PRISMA для проведенного исследования

Б) Критерии включения/исключения.

В мета-анализ были включены исследования, соответствующие следующим критериям:

1. Проспективное рандомизированное контролируемое исследование (РКИ).

2. Сравнение эффективности ранней и отложенной лапароскопической холецистэктомии в лечении острого холецистита у взрослых.

3. Доступность информации как минимум об одном из результатов исследования (общее количество осложнений, количество ятрогенных повреждений желчных протоков, общее время нахождения в больнице, общее время операции, количество переводов операции в лапаротомию, общие затраты на лечение).

4. Ранняя лапароскопическая холецистэктомия выполнена в течение 7 дней от появления симптомов, отложенная лапароскопическая холецистэктомия выполнена после консервативного лечения, проводимого в течение не менее 7 дней. 
Таблица 1.

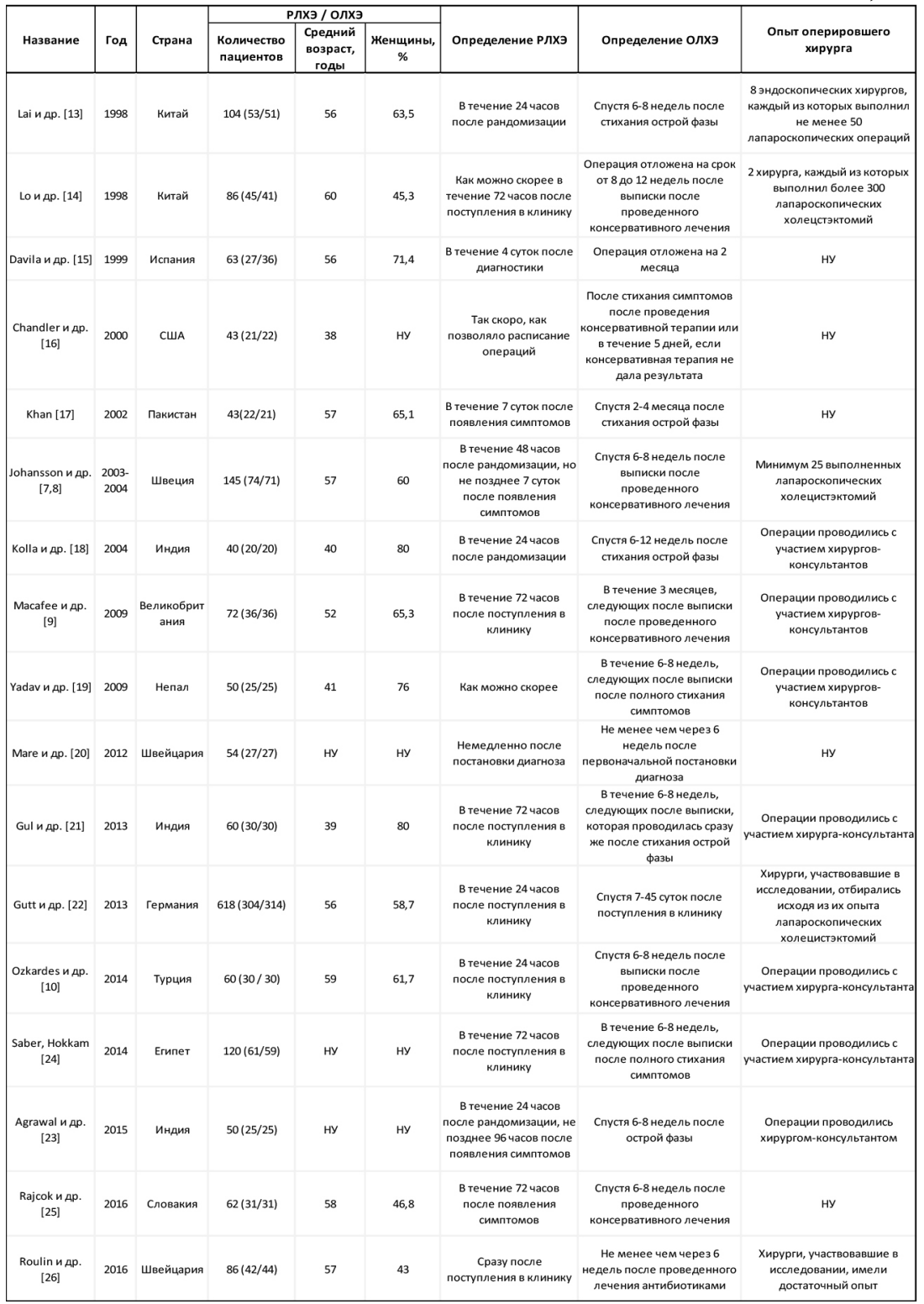


Исключены:

1. Ретроспективные и нерандомизированные исследования.

2. Исследования, выполняющие сравнение эффективности холецистэктомии и других методов лечения холецистита.

3. Исследования, целевая группа в которых - больные хроническим холециститом.

В) Исходы лечения.

Исходом, представлявшим наибольшее значение, был показатель общего количества осложнений в обеих исследуемых группах. Вторично исследовались показатели общего времени нахождения в больнице, общего времени операции, количество переводов операции в лапаротомию, количество ятрогенных повреждений желчных протоков, а также общие затраты на лечение.

Д) Оценка методологического качества включенных исследований.

В исследование были включены 17 исследований, по их данным была составлена Таблица №1, включающая общие характеристики исследования и групп, изучавшихся в нем. Общее количество пациентов - 1756 человек, из которых в группу ранней лапароскопической холецистэктомии попали 873 человека, а в группу отложенной лапароскопической холецистэктомии - 883 человека. Для каждого РКИ также описаны определения ранней и отложенной лапароскопических холецистэктомий, приведенные авторами, а также оценка опыта хирурга, проводившего операции.

Оценка риска системных ошибок в исследованиях проводилась в соответствие с рекомендациями Кокрейновского сотрудничества (Cochrane Collaboration), изложенными в Кокрейновском руководстве по систематическим обзорам медицинских вмешательств (Cochrane Handbook for Systematic Reviews of Interventions) и Кокрейновском групповом гепатобилиарном модуле (Cochrane Hepato-Biliary group module), её результаты приведены в Таблице №2.

Таблииа 2.

\begin{tabular}{|c|c|c|c|c|c|c|c|}
\hline Название & $\begin{array}{c}\text { Ран- } \\
\text { доми- } \\
\text { зация }\end{array}$ & $\begin{array}{c}\text { Со- } \\
\text { крытие } \\
\text { процесса } \\
\text { рандоми- } \\
\text { зации }\end{array}$ & $\begin{array}{c}\text { Маскировка } \\
\text { данных для } \\
\text { пациента и } \\
\text { медперсо- } \\
\text { нала }\end{array}$ & $\begin{array}{c}\text { Маски- } \\
\text { ровка } \\
\text { оценки } \\
\text { исхода }\end{array}$ & $\begin{array}{c}\text { Непол- } \\
\text { ные } \\
\text { результа- } \\
\text { тивные } \\
\text { данные }\end{array}$ & $\begin{array}{c}\text { Выбо- } \\
\text { рочная } \\
\text { отчет- } \\
\text { ность }\end{array}$ & $\begin{array}{c}\text { Сопоста- } \\
\text { вимость } \\
\text { исхдных } \\
\text { характе- } \\
\text { ристик }\end{array}$ \\
\hline Lai и др. & + & + & $?$ & $?$ & + & + & + \\
\hline Lo и др. & + & + & $?$ & $?$ & + & + & + \\
\hline Davila и др. & $?$ & $?$ & $?$ & $?$ & $?$ & + & $?$ \\
\hline Chandler и др. & $?$ & $?$ & $?$ & $?$ & $?$ & + & + \\
\hline Khan & $?$ & $?$ & $?$ & $?$ & $?$ & + & + \\
\hline
\end{tabular}


Окончание табл. 2.

\begin{tabular}{|c|c|c|c|c|c|c|c|}
\hline Johansson и др. & + & + & $?$ & $?$ & + & + & + \\
\hline Kolla и др. & + & + & $?$ & $?$ & + & + & + \\
\hline Macafeе и др. & + & + & - & - & + & - & + \\
\hline Yadav и др. & $?$ & $?$ & $?$ & $?$ & $?$ & - & + \\
\hline Mare и др. & $?$ & $?$ & - & - & + & + & $?$ \\
\hline Gul и др. & + & + & $?$ & $?$ & $?$ & + & $?$ \\
\hline Gutt и др. & + & + & $?$ & $?$ & + & + & + \\
\hline Ozkardes и др. & + & + & $?$ & $?$ & + & + & + \\
\hline Agrawal и др. & $?$ & $?$ & $?$ & $?$ & + & + & + \\
\hline Saber/Ноkkаm & + & + & $?$ & $?$ & + & - & + \\
\hline Rajcok и др. & $?$ & $?$ & $?$ & $?$ & + & + & + \\
\hline Roulin и др. & + & + & - & - & + & + & + \\
\hline
\end{tabular}

Примечание: «+» - низкий риск системной ошибки, «?»- неясный риск системной ошибки, «-» - высокий риск системной ошибки.

Для каждого исследования риск системной ошибки оценивался по 7 критериям (результат, соответствующий низкому риску системной ошибки указан в скобках): 1) генерация случайной последовательности (метод рандомизации указан и выбран верно), 2) сокрытие процесса рандомизации (метод выбран верно и не вызывает искажения результатов оценки значимости эффекта), 3) маскировка данных («слепой» метод) о выбранном типе лечения для пациента и медицинского персонала (во всех исследованиях риск ошибки высокий, так как метод невозможно применить для пациентов и хирургов, выполняющих операцию), 4) маскировка («слепой» метод) оценки исхода (маскировка была применена и способ ее проведения описан), 5) неполные данные об исходе (отсутствующие данные не вызывают искажения результатов оценки значимости эффекта или применен эффективный метод замены отсутствующих данных), 6) выборочная отчетность (важные исходы, которые однозначно регистрировались в ходе исследования - осложненность, смертность и т.д. - описаны в результатах исследования), 7) сопоставимость данных исходных характеристик (исходные характеристики обеих групп сопоставимы между собой). Неясный риск системной ошибки регистрировался, если необходимая для оценки информация отсутствовала или была недостаточной.

Все исследования имеют высокий риск методологической ошибки, так как ни в одном из них не была использована маскировка данных о выбранном типе лечения для пациента и медицинского персонала, а также маскировка оценки исхода. Однако, учитывая невозможность использования 
данного метода в условиях подобных клинических исследований по этическим соображениям, методологическое качество признается приемлемым.

Е) Синтез количественных данных.

Для дихотомических исходов рассчитывался относительный риск с 95\% доверительным интервалом (ДИ), для исходов, учитывающих временной интервал (общее время нахождения в больнице, общее время операции) рассчитывалось средние различия в длительности временных интервалов между группами с 95\% ДИ. Для тех исследований, в которых не было представлено стандартного отклонения, а средние значения заменены медианными, расчеты в случае исходов, учитывающих временной интервал не проводились, чтобы избежать искажения результатов.

Статистическая неоднородность измерялась по показателю $\mathrm{I}^{2}$ : при его значении от 0 до $25 \%$ гетерогенность признавалась низкой, от 25 до $50 \%$ умеренной, выше 50\% - высокой. Для тех исходов, в которых гетерогенность исследований была высокой использовалась модель случайных эффектов, взвешенная по методу Мантеля-Хэнзеля. Модель фиксированных эффектов, взвешенная по методу Мантеля-Хэнзеля применялась при низкой и умеренной гетерогенности. Гетерогенность оценивалась по показателю $\mathrm{I}^{2}$, для визуальной оценки был построен так называемы «лесовидный» график. Весь статистический анализ проводился при помощи программы RevMan 5.3, рекомендованной Кокрейновским сотрудничеством. Результаты считались статистически значимыми при р>0,05.

Ж) Последовательный статистический анализ.

Последовательный статистический анализ для всех этапов мета-анализа был выполнен с помощью программы TSA (Trial Sequential Analysis), разработанной Копенгагенским исследовательским подразделением (Copenhagen Trial Unit).

\section{Результаты}

Среди всех исследований летальные случаи наблюдались только у Gutt и др. [22], при этом различий между исследуемыми группами не было. В 8 исследованиях данных о смертности не было представлено (Ozkardes и др. [10], Rajcok и др. [25], Roulin и др. [26], Yadav и др. [19], Macafee и др. [9], Gul и др. [21], Khan и др. [17], Мare и др. [20]).

Ятрогенное повреждение желчных протоков оценивалось отдельно изза своей высокой клинической значимости. Значимых различий в частоте возникновения этого осложнения между группами ранней и отложенной лапароскопической холецистэктомии не наблюдалось (Отношение шансов 
0,66; 95\% ДИ 0,11, 3,80). Из-за того, что данное осложнение встречается менее чем у $1 \%$ пациентов, эффект оценивался отношением шансов по методу Пето. Гетерогенность исследований низкая I ${ }^{2}=18 \%$, поэтому применялась модель фиксированных эффектов.

Таблицуа 3.

\begin{tabular}{|c|c|c|c|c|c|c|c|}
\hline \multirow{2}{*}{$\begin{array}{c}\text { Исследование } \\
\text { или подгруппа }\end{array}$} & \multicolumn{2}{|c|}{ РЛХЭ } & \multicolumn{2}{|c|}{ ОЛХЭ } & \multirow{2}{*}{ Bec } & \multirow{2}{*}{$\begin{array}{c}\text { ОШ Пето, } \\
\text { фиксированный, } \\
\text { 95\% ДИ } \\
\end{array}$} & \multirow{2}{*}{ Год } \\
\hline & События & Всего & События & Всего & & & \\
\hline Lо и др. [14] & 0 & 45 & 1 & 41 & $20,00 \%$ & $0,12[0,00,6,21]$ & 1998 \\
\hline Lai и др. [13] & 0 & 53 & 0 & 51 & & Не оцениваемо & 1998 \\
\hline Davila и др. [15] & 0 & 27 & 0 & 36 & & Не оцениваемо & 1999 \\
\hline Kolla и др. [18] & 1 & 20 & 0 & 20 & $20,00 \%$ & $7,39[0,15,372,38]$ & 2004 \\
\hline Johansson и др. $[7,8]$ & 0 & 74 & 1 & 71 & $20,00 \%$ & $0,13[0,00,6,54]$ & 2004 \\
\hline Gul и др. [21] & 0 & 30 & 0 & 30 & & Не оцениваемо & 2013 \\
\hline Ozkardes и др. [10] & 1 & 30 & 0 & 30 & $20,00 \%$ & $7,39[0,15,372,38]$ & 2014 \\
\hline Agrawal и др. [23] & 0 & 25 & 0 & 25 & & Не оцениваемо & 2015 \\
\hline Rajcok и др. [25] & 0 & 31 & 0 & 31 & & Не оцениваемо & 2016 \\
\hline Roulin и др. [26] & 0 & 42 & 1 & 44 & $20,00 \%$ & $0,14[0,00,7,15]$ & 2016 \\
\hline Всего (95\% ДИ): & & 377 & & 379 & $100,00 \%$ & $0,66[0,11,3,80]$ & \\
\hline Всего событий: & 2 & & 3 & & & & \\
\hline Гетерогенность: & \multicolumn{4}{|c|}{$\mathrm{Chi}^{2}=4,88, \mathrm{df}=4(\mathrm{P}=0,30) ; \mathrm{I}^{2}=18 \%$} & & & \\
\hline $\begin{array}{l}\text { Тест на суммар- } \\
\text { ный эффект: }\end{array}$ & $\mathrm{Z}=0,47(\mathrm{P}=$ & $=0,64)$ & & & & & \\
\hline
\end{tabular}

Примечание: РЛХЭ - группа ранней лапароскопической холецистэктомии, ОЛХЭ - группа отложенной лапароскопической холецистэктомии.

При проведении последовательного статистического анализа (Приложение №2) было обнаружено, что для получения достоверных результатов размер выборки должен быть большим (n=100574 человек), а в рамках данного мета-анализа количество пациентов составило менее $1 \%$ от необходимого объема, граница значимости также не пересечена.

Информация об общем количестве осложнений (интра- и постоперативных) была представлена в 17 исследованиях. Различий между двумя группами по общему количеству осложнений не наблюдалось (Относительный риск 0,$92 ; 95 \%$ ДИ $0,61,1,38)$. Гетерогенность исследований высокая $\mathrm{I}^{2}=72 \%$, применялась модель случайных эффектов. Последовательный статистический анализ показал, что размер выборки не достиг должных значений (n=6259), а границы значимости пересечены не были. 
Таблицуа 4.

\begin{tabular}{|c|c|c|c|c|c|c|c|}
\hline \multirow{2}{*}{$\begin{array}{c}\text { Исследование } \\
\text { или подгруппа }\end{array}$} & \multicolumn{2}{|c|}{ РЛХЭ } & \multicolumn{2}{|c|}{ ОЛХЭ } & \multirow{2}{*}{ Bec } & \multirow{2}{*}{$\begin{array}{c}\text { ОР М-Х, случай- } \\
\text { ный, 95\% ДИ }\end{array}$} & \multirow{2}{*}{ Год } \\
\hline & События & Всего & События & Всего & & & \\
\hline Lai и др. [13] & 5 & 53 & 3 & 51 & $4,60 \%$ & $1,60[0,40,6,37]$ & 1998 \\
\hline Lо и др. [14] & 6 & 45 & 12 & 41 & $6,80 \%$ & $0,46[0,19,1,10]$ & 1998 \\
\hline Davila и др. [15] & 5 & 27 & 13 & 36 & $6,70 \%$ & $0,51[0,21,1,26]$ & 1999 \\
\hline Chandler и др. [16] & 2 & 21 & 2 & 22 & $3,20 \%$ & $1,05[0,16,6,77]$ & 2000 \\
\hline Khan [17] & 3 & 22 & 3 & 21 & $4,30 \%$ & $0,95[0,22,4,21]$ & 2002 \\
\hline Kolla и др. [18] & 4 & 20 & 3 & 20 & $4,70 \%$ & $1,33[0,34,5,21]$ & 2004 \\
\hline Johansson и др. [7,8] & 13 & 74 & 7 & 71 & $6,90 \%$ & $1,78[0,75,4,21]$ & 2004 \\
\hline Yadav и др. [19] & 16 & 25 & 15 & 25 & $8,90 \%$ & $1,07[0,69,1,65]$ & 2009 \\
\hline Macafee и др. [9] & 8 & 36 & 4 & 36 & $5,70 \%$ & $2,00[0,66,6,06]$ & 2009 \\
\hline Mare и др. [20] & 6 & 27 & 13 & 27 & $7,20 \%$ & $0,46[0,21,1,03]$ & 2012 \\
\hline Gul и др. [21] & 6 & 30 & 4 & 30 & $5,50 \%$ & $1,50[0,47,4,78]$ & 2013 \\
\hline Gutt и др. [22] & 43 & 304 & 127 & 314 & $9,40 \%$ & $0,35[0,26,0,48]$ & 2013 \\
\hline Ozkardes и др. [10] & 8 & 30 & 0 & 30 & $1,70 \%$ & $17,00[1,03,281,91]$ & 2014 \\
\hline Saber, Hokkam [24] & 16 & 61 & 10 & 59 & $7,70 \%$ & $1,55[0,77,3,13]$ & 2014 \\
\hline Agrawal и др. [23] & 8 & 25 & 2 & 25 & $4,40 \%$ & $4,00[0,94,17,00]$ & 2015 \\
\hline Rajcok и др. [25] & 6 & 42 & 17 & 44 & $7,00 \%$ & $0,37[0,16,0,85]$ & 2016 \\
\hline Roulin и др. [26] & 3 & 31 & 8 & 31 & $5,20 \%$ & $0,38[0,11,1,28]$ & 2016 \\
\hline Всего (95\% ДИ): & & 873 & & 883 & $100,00 \%$ & $0,92[0,61,1,38]$ & \\
\hline Всего событий: & 158 & & 243 & & & & \\
\hline Гетерогенность: & \multicolumn{6}{|c|}{$\mathrm{Tau}^{2}=0,43 ; \mathrm{Chi}^{2}=56,17, \mathrm{df}=16(\mathrm{P}<0,00001) ; \mathrm{I}^{2}=72 \%$} & \\
\hline $\begin{array}{c}\text { Тест на суммарный } \\
\text { эффект: }\end{array}$ & $\mathrm{Z}=0,42(\mathrm{P}$ & $=0,0$ & & & & & \\
\hline
\end{tabular}

Примечание: РЛХЭ - группа ранней лапароскопической холецистэктомии, ОЛХЭ - группа отложенной лапароскопической холецистэктомии.

Информация о количество переводов лапароскопических операций в лапаротомические была представлена в 14 исследованиях. Различий между группами ранней и отложенной лапароскопической холецистэктомии не наблюдалось (Относительный риск 0,98; 95\% ДИ 0,76, 1,27), гетерогенность была низкой $=0 \%$. Последовательный статистический анализ показал, что размер выборки не достиг должных значений (n=5155), а границы значимости пересечены не были.

Информация об общем количество койко-дней была была изложена в 5 исследованиях (Lai и др. [13]; Kolla и др. [18]; Ozkardes и др. [10]; Saber/ Hokkam [24]; Yadav и др. [19]). Длительность нахождения в стационаре была ниже в группе ранней лапароскопической холецистэктомии на 3 дня 
(Разность средних -3,10; 95\% ДИ -3,51,-2,69), гетерогенность исследований умеренная $\left(\mathrm{I}^{2}=26 \%\right)$.

Таблицуа 5.

\begin{tabular}{|c|c|c|c|c|c|c|c|}
\hline \multirow{2}{*}{$\begin{array}{c}\text { Исследование } \\
\text { или подгруппа }\end{array}$} & \multicolumn{2}{|c|}{ РЛХЭ } & \multicolumn{2}{|c|}{ ОЛХЭ } & \multirow[b]{2}{*}{ Bec } & \multirow{2}{*}{$\begin{array}{c}\text { ОР М-Х, } \\
\text { фиксированный, } \\
95 \% \text { ДИ }\end{array}$} & \multirow[b]{2}{*}{ Год } \\
\hline & События & Всего & События & Всего & & & \\
\hline Lai и др. [13] & 11 & 53 & 9 & 51 & $9,30 \%$ & $1,18[0,53,2,60]$ & 1998 \\
\hline Lо и др. [14] & 5 & 45 & 9 & 41 & $9,60 \%$ & $0,51[0,18,1,39]$ & 1998 \\
\hline Davila и др. [15] & 1 & 27 & 6 & 36 & $5,20 \%$ & $0,22[0,03,1,74]$ & 1999 \\
\hline Johansson и др. $[7,8]$ & 23 & 74 & 20 & 71 & $20,70 \%$ & $1,10[0,67,1,82]$ & 2004 \\
\hline Kolla и др. [18] & 5 & 20 & 5 & 20 & $5,10 \%$ & $1,00[0,34,2,93]$ & 2004 \\
\hline Yadav и др. [19] & 4 & 25 & 3 & 25 & $3,00 \%$ & $1,33[0,33,5,36]$ & 2009 \\
\hline Macafee и др. [9] & 1 & 36 & 1 & 36 & $1,00 \%$ & $1,00[0,07,15,38]$ & 2009 \\
\hline Gul и др. [21] & 3 & 30 & 4 & 30 & $4,10 \%$ & $0,75[0,18,3,07]$ & 2013 \\
\hline Gutt и др. [22] & 30 & 304 & 33 & 314 & $32,90 \%$ & $0,94[0,59,1,50]$ & 2013 \\
\hline Saber, Hokkam [24] & 3 & 61 & 1 & 59 & $1,00 \%$ & $2,90[0,31,27,11]$ & 2014 \\
\hline Ozkardes и др. [10] & 4 & 30 & 0 & 30 & $0,50 \%$ & $9,00[0,51,160,17]$ & 2014 \\
\hline Agrawal и др. [23] & 4 & 25 & 2 & 25 & $2,00 \%$ & $2,00[0,40,9,95]$ & 2015 \\
\hline Rajcok и др. [25] & 1 & 42 & 0 & 44 & $0,50 \%$ & $3,14[0,13,74,98]$ & 2016 \\
\hline Roulin и др. [26] & 1 & 31 & 5 & 31 & $5,10 \%$ & $0,20[0,02,1,61]$ & 2016 \\
\hline Всего (95\% ДИ): & & 803 & & 813 & $100,00 \%$ & $0,98[0,76,1,27]$ & \\
\hline Всего событий: & 96 & & 98 & & & & \\
\hline Гетерогенность: & \multicolumn{5}{|c|}{$\mathrm{Chi}^{2}=11,11, \mathrm{df}=13(\mathrm{P}=0,60) ; \mathrm{I}^{2}=0 \%$} & & \\
\hline $\begin{array}{l}\text { Тест на суммар- } \\
\text { ный эффект: }\end{array}$ & $\mathrm{Z}=0,15(\mathrm{P}$ & $=0,88)$ & & & & & \\
\hline
\end{tabular}

Примечание: РЛХЭ - группа ранней лапароскопической холецистэктомии, ОЛХЭ - группа отложенной лапароскопической холецистэктомии.

Информация из исследований, в которых отсутствовали данные о средних величинах и стандартном отклонении, а также были заменены медианными величинами, не была включена в мета-анализ во избежание искажения результатов исследования в соответствии с рекомендациями Кокрейновского сотрудничества. При проведении последовательного статистического анализа, было выявлено, что размер выборки достиг необходимых значений $(\mathrm{n}=172)$, границы значимости также пересечены, что позволяет говорить о значимом свидетельстве меньшей продолжительности нахождения в стационаре пациентов группы ранней лапароскопической холецистэктомии. 
Таблииа 6.

\begin{tabular}{|c|c|c|c|c|c|}
\hline $\begin{array}{l}\text { Исследование } \\
\text { или подгруппа }\end{array}$ & $\begin{array}{l}\text { Разность } \\
\text { средних }\end{array}$ & $\begin{array}{c}\text { Стандартная } \\
\text { ошибка }\end{array}$ & Bec & $\begin{array}{c}\text { Разность средних } \\
\text { М-Х, фиксирован- } \\
\text { ный, 95\% ДИ }\end{array}$ & Год \\
\hline Lai и др. [13] & -4 & 0,6864 & $9,20 \%$ & $-4,00[-5,35,-2,65]$ & 1998 \\
\hline Kolla и др. [18] & -6 & 2,3576 & $0,80 \%$ & $-6,00[-10,62,-1,38]$ & 2004 \\
\hline Yadav и др. [19] & $-2,9$ & 0,4377 & $22,60 \%$ & $-2,90[-3,76,-2,04]$ & 2009 \\
\hline Saber, Hokkam [24] & $-3,3$ & 0,3309 & $39,60 \%$ & $-3,30[-3,95,-2,65]$ & 2014 \\
\hline Ozkardes и др. [10] & $-2,6$ & 0,3951 & $27,80 \%$ & $-2,60[-3,37,-1,83]$ & 2014 \\
\hline Всего (95\% ДИ): & & & $100,00 \%$ & $-3,10[-3,51,-2,69]$ & \\
\hline Гетерогенность: & \multicolumn{3}{|c|}{$\mathrm{Chi}^{2}=5,41, \mathrm{df}=4(\mathrm{P}=0,25) ; \mathrm{I}^{2}=26 \%$} & & \\
\hline $\begin{array}{c}\text { Тест на суммарный } \\
\text { эффект: }\end{array}$ & \multicolumn{2}{|c|}{$Z=14,89(P<0,00001)$} & & & \\
\hline
\end{tabular}

Примечание: РЛХЭ - группа ранней лапароскопической холецистэктомии, ОЛХЭ - группа отложенной лапароскопической холецистэктомии.

\begin{tabular}{|c|c|c|c|}
\hline $\begin{array}{l}\text { Исследование или } \\
\text { подгруппа }\end{array}$ & $\begin{array}{c}\text { Разность средних } \\
\text { ОД, фиксированный, } 95 \% \text { ДИ }\end{array}$ & \multicolumn{2}{|c|}{$\begin{array}{c}\text { Разность средних } \\
\text { од, фиксированный, } 95 \% \text { ДИ }\end{array}$} \\
\hline Lai и др. & $-4.00[-5.35,-2.65]$ & $\longrightarrow$ & \\
\hline Kolla и др. & $-6.00[-10.62,-1.38]$ & & \\
\hline Yadav и др. & $-2.90[-3.76,-2.04]$ & $\rightarrow-$ & \\
\hline Ozkardes и дp. & $-2.60[-3.37,-1.83]$ & $\rightarrow-$ & \\
\hline Saber/Hokkam & $-3.30[-3.95,-2.65]$ & +2 & \\
\hline \multirow[t]{3}{*}{ Всего (95\% Ди) } & $-3.10[-3.51,-2.69]$ & $\uparrow$ & \\
\hline & & $-10 \quad-5$ & 10 \\
\hline & & В пользу РЛХЭ & В пользу олХЕ \\
\hline
\end{tabular}

Рис. 2. Лесовидный график оценки среднего количества койко-дней

Информация об общем времени операции была представлена в 4 исследованиях (Lai и др. [13]; Kolla и др. [18]; Yadav и др. [19]; Ozkardes и др. [10]). Значимых различий между группами ранней и отложенной лапароскопической холецистэктомии не было найдено (Разность средних 13,99; $95 \%$ ДИ -3,24, 31,22), гетерогенность исследований высокая $\left(I^{2}=77 \%\right)$. Информация из исследований, в которых данные о средних величинах и стандартном отклонении отсутствовали или были заменены медианными величинами, не была включена в мета-анализ во избежание искажения результатов исследования в соответствии с рекомендациями Кокрейновского сотрудничества. Последовательный статистический анализ показал, что размер выборки не достиг должных значений $(\mathrm{n}=869)$, границы значимости также не пересечены. 
Таблииа 7.

\begin{tabular}{|c|c|c|c|c|c|}
\hline $\begin{array}{c}\text { Исследование } \\
\text { или подгруппа }\end{array}$ & $\begin{array}{l}\text { Разность } \\
\text { средних }\end{array}$ & \begin{tabular}{|c|}
$\begin{array}{c}\text { Стандартная } \\
\text { ошибка }\end{array}$ \\
\end{tabular} & Bec & \begin{tabular}{|c|} 
Разность средних М-Х, \\
случайный, 95\% ДИ \\
\end{tabular} & Год \\
\hline Lai и др. [13] & 16,2 & 7,1926 & $27,60 \%$ & $16,20[2,10,30,30]$ & 1998 \\
\hline Kolla и др. [18] & 11,3 & 14,2144 & $18,00 \%$ & $11,30[-16,56,39,16]$ & 2004 \\
\hline Yadav и др. [19] & 33,1 & 8,0872 & $26,30 \%$ & $33,10[17,25,48,95]$ & 2009 \\
\hline Ozkardes и др. [10] & $-4,33$ & 6,8143 & $28,10 \%$ & $-4,33[-17,69,9,03]$ & 2014 \\
\hline Всего (95\% ДИ): & & & $100,00 \%$ & $13,99[-3,24,31,22]$ & \\
\hline Гетерогенность: & \multicolumn{4}{|c|}{$\mathrm{Tau}^{2}=100,27 ; \mathrm{Chi}^{2}=13,92, \mathrm{df}=4(\mathrm{P}=0,008) ; \mathrm{I}^{2}=71 \%$} & \\
\hline $\begin{array}{c}\text { Tест на суммарный } \\
\text { эффект: }\end{array}$ & $Z=2,6$ & $8(\mathrm{P}=0,007)$ & & & \\
\hline
\end{tabular}

Примечание: РЛХЭ - группа ранней лапароскопической холецистэктомии, ОЛХЭ - группа отложенной лапароскопической холецистэктомии.

В четырех исследованиях были представлены данные о затратах на лечение, при этом в исследовании Ozkardes и др. [10] данные о затратах были представлены в турецких лирах, а в исследовании Macafee и др. [9] - в фунтах стерлингов. Затраты были переведены в общую для всех исследований валюту - евро по курсу на 18 августа 2018 года. Затраты были меньше в группе ранней лапароскопической холецистэктомии (Стандартизованная разность средних -0,78; 95\% ДИ -1,31; -0,26), гетерогенность исследований высокая $\left(\mathrm{I}^{2}=86 \%\right)$. Последовательный статистический анализ показал, что размер выборки достиг должных значений $(\mathrm{n}=564)$, границы значимости также были пересечены, что служит подтверждением значимого снижения затрат на лечение пациентов при применении ранней лапароскопической холецистэктомии.

Таблицуа 8.

\begin{tabular}{|c|c|c|c|c|c|c|c|}
\hline \multirow{2}{*}{$\begin{array}{c}\text { Исследование } \\
\text { или подгруппа }\end{array}$} & \multicolumn{2}{|c|}{ РЛХЭ } & \multicolumn{2}{|c|}{ ОЛХЭ } & \multirow{2}{*}{ Bec } & \multirow{2}{*}{\begin{tabular}{|c|} 
Стандартизованная \\
разность средних ОД, \\
случайный, 95\% ДИ \\
\end{tabular}} & \multirow{2}{*}{ Год } \\
\hline & Среднее & SD & Среднее & SD & & & \\
\hline Macafee и др. [9] & 6620,3 & 2738 & 6867,8 & 3633,3 & $24,40 \%$ & $-0,08[-0,54,0,39]$ & 2009 \\
\hline Gutt и др. [22] & 2919 & 951,8 & 4262 & 2102 & $29,20 \%$ & $-0,82[-0,98,-0,65]$ & 2013 \\
\hline $\begin{array}{c}\text { Ozkardes и др. } \\
{[10]} \\
\end{array}$ & 375,1 & 113,3 & 557 & 77,6 & $21,40 \%$ & $-1,85[-2,46,-1,24]$ & 2014 \\
\hline Roulin и др. [26] & 9349 & 5417,7 & 12361 & 5922,5 & $25,00 \%$ & $-0,53[-0,96,-0,09]$ & 2016 \\
\hline Всего (95\% ДИ): & & & & & $100,00 \%$ & $-0,78[-1,31,-0,26]$ & \\
\hline Гетерогенность: & \multicolumn{6}{|c|}{$\mathrm{Tau}^{2}=0,24 ; \mathrm{Chi}^{2}=22,21, \mathrm{df}=3(\mathrm{P}<0,0001) ; \mathrm{I}^{2}=86 \%$} & \\
\hline $\begin{array}{c}\text { Тест на суммар- } \\
\text { ный эффект: }\end{array}$ & $Z=2,93$ & $(\mathrm{P}=0,00$ & & & & & \\
\hline
\end{tabular}

Примечание: РЛХЭ - группа ранней лапароскопической холецистэктомии, ОЛХЭ - группа отложенной лапароскопической холецистэктомии. 


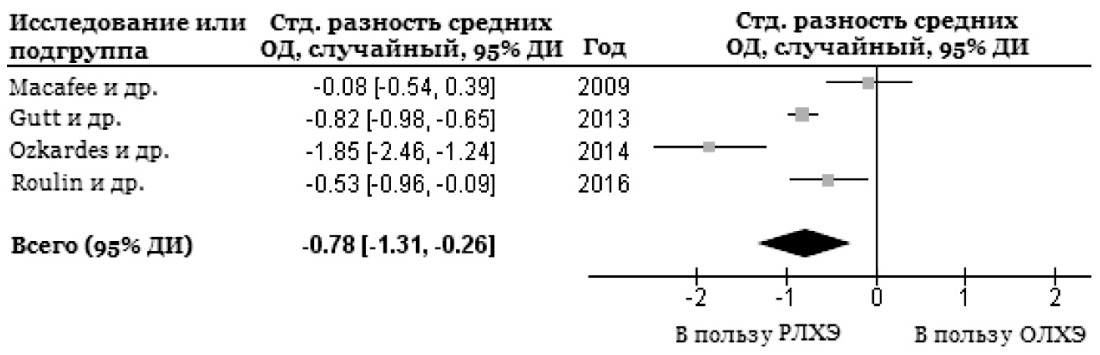

\section{Обсуждение результатов}

В ходе данного мета-анализа было выявлено отсутствие различий между группами ранней и отложенной лапароскопической холецистэктомии как по общему количеству осложнений, так и по количеству переводов лапароскопической операции в лапаротомическую, и по количеству ятрогенного повреждения желчных протоков. Такие данные могут свидетельствовать о том, что и ранняя, и отложенная лапароскопическая холецистэктомия не различаются по рискам для пациента. Отдельного упоминания стоят данные последовательно статистического анализа, которые для всех дихотомических исходов показали недостаточный размер выборки. Это говорит о необходимости проведения исследований с более крупным размером выборки для получения более точных сведений о различиях между группами.

Данные о смертности были изложены только в 9 из 17 попавших в мета-анализ исследований, при этом летальные случаи были только в одном, поэтому провести мета-анализ по данному критерию не представлялось возможным. Для уточнения различий в смертности между группами ранней и отложенной лапароскопической холецистэктомии также требуются более крупные исследования.

Наблюдалось снижение среднего количества койко-дней для пациентов из группы ранней лапароскопической холецистэктомии на 3 дня, при этом значимость различий была подтверждена в ходе последовательного статистического анализа. Эти данные имеют высокую клиническую ценность, так как меньшее количество дней, проведенных пациентом в больнице способствуют снижению загруженности больниц и медперсонала.

При исследовании среднего оперативного времени не было найдено статистически значимых различий, таким образом более раннее проведение оперативного вмешательства не влияет на его длительность. 
Затраты на лечение были значительно меньше в группе ранней лапароскопической холецистэктомии, что также было подтверждено последовательным статистическим анализом. Однако требуется более подробное исследование затрат на лечение, так как гетерогенность исследований была высокой.

Меньшая стоимость лечения и меньшее количество койко-дней у пациентов, которым выполнили холецистэктомию в течение первой недели после появления симптомов, являются высоко значимыми для процесса организации здравоохранения. Уменьшение издержек в пересчете на каждого больного означает повышение КПД работы медучреждений и качества оказания медицинской помощи. По оценкам исследований, в Великобритании использование ранней лапароскопической холецистэктомии в качестве основного метода лечения острого холецистита может сэкономить до 8,5 миллионов фунтов стерлингов ежегодно [27].

Возможно, ранняя холецистэктомия требует большей квалификации хирургов, так как является более сложной операцией в техническом плане и несет дополнительные риски ятрогенного повреждения желчных протоков, что, однако, не нашло подтверждения в ходе данного мета-анализа.

Таким образом, при повсеместном введении ранней холецистэктомии в качестве основного способа лечения острого холецистита, можно добиться заметного снижения времени, проведенного пациентами в больнице, и средств, потраченных на лечение. В рамках данного мета-анализа не было обнаружено различий в количестве осложнений между группами, однако все операции выполнялись опытными хирургами, а основные риски возникновения ятрогенных осложнений возникают при проведении операции малоопытными специалистами.

\section{Заключение}

Применение раннего оперативного вмешательства для лечения острого холецистита (в течение первой недели после появления симптомов) значительно сокращает количество времени и средств, затраченных на лечение пациента, не создавая повышенного риска возникновения любых интра- и постоперационных осложнений.

Информация о конфликте интересов. Автор заявляет об отсутствии конфликта интересов.

Информация о спонсорстве. Автор заявляет об отсутствии стороннего финансирования. 


\section{Список литературы}

1. Everhart J.E., Ruhl C.E. Burden of digestive diseases in the United States. Part III: liver, biliary tract and pancreas // Gastroenterology, 2009, 136, pp. 1134-1144.

2. Aerts R., Penninckx F. The burden of gallstone disease in Europe // Aliment. Pharmacol. Ther., 2003, 18 (Suppl. 3), pp. 49-53.

3. Воротынцев А. С. Современные представления о диагностике и лечении желчнокаменной болезни и хронического калькулезного холецистита // Лечащий врач. 2012. №2. С. 54-58.

4. Indar A.A., Beckingham I.J. Acute cholecystitis // BMJ: British Medical Journal, 2002, 325 (7365), pp. 639-643.

5. Бебуришвили А.Г., Прудков М.И., Совцов С.А. и др. Национальные клинические рекомендации «Острый холецистит». Труды ХІІ Съезда хирургов России «Актуальные вопросы хирургии», г. Ростов-на-Дону. 2015.

6. Yamashita Y., Takada T., Hirata K. A survey of the timing and approach to the surgical management of patients with acute cholecystitis in Japanese hospitals // J. Hepatobiliary Pancreat. Surg., 2006, 13, pp. 409-415.

7. Johansson M., Thune A., Blomqvist A., Nelvin L., Lundell L. Management of acute cholecystitis in the laparoscopic era: results of a prospective, randomized clinical trial // J. Gastrointest. Surg., 2003, 7, pp. 642-645.

8. Johansson M., Thune A., Blomqvist A, Nelvin L., Lundell L. Impact of choice of therapeutic strategy for acute cholecystitis on patient's health-related quality of life. Results of a randomized, controlled clinical trial // Dig. Surg., 2004, №21, pp. 359-362.

9. Macafee D.A., Humes D.J., Bouliotis G., Beckingham I.J., Whynes D.K., Lobo D.N. Prospective randomized trial using cost-utility analysis of early versus delayed laparoscopic cholecystectomy for acute gallbladder disease // Br. J. Surg., 2009, 96, pp. 1031-1040.

10. Ozkardes A.B., Tokaç M., Dumlu E.G., Bozkurt B., Ciftçi A.B., Yeti, sir F. et al. Early versus delayed laparoscopic cholecystectomy for acute cholecystitis: a prospective, randomized study // Int. Surg., 2014, 99, pp. 56-61.

11. Zhou M.W., Gu X.D., Xiang J.B., Chen Z.Y. Comparison of clinical safety and outcomes of early versus delayed laparoscopic cholecystectomy for acute cholecystitis: a meta-analysis // ScientificWorldJournal, 2014, 2014:274516.

12. Menahem B., Mulliri A., Fohlen A. et al. Delayed laparoscopic cholecystectomy increases the total hospital stay compared to an early laparoscopic cholecystectomy after acute cholecystitis: an updated meta-analysis of randomized controlled trials // HPB (Oxford), 2015, 17(10), pp. 857-862. 
13. Lai P.B., Kwong K.H., Leung K.L. et al. Randomized trial of early versus delayed laparoscopic cholecystectomy for acute cholecystitis // Br. J. Surg., 1998, 85, pp. 764-767.

14. Lo C.M., Liu C.L., Fan S.T. et al. Prospective randomized study of early versus delayed laparoscopic cholecystectomy for acute cholecystitis // Ann. Surg., 1998, 227, pp. 461-467.

15. Dávila D., Manzanares C., Picho M.L. et al. Experience in the treatment (early vs. delayed) of acute cholecystitis via laparoscopy // Cirugia Española, 1999, 66(Suppl 1), pp. 233.

16. Chandler C.F., Lane J.S., Ferguson P. et al. Prospective evaluation of early versus delayed laparoscopic cholecystectomy for treatment of acute cholecystitis // Am. Surg., 2000, 66, pp. 896-900.

17. Khan S.S.A. Early versus delayed cholecystectomy for acute cholecystitis, a prospective randomized study // Pak. J. Gastroenterol., 2002, 16, pp. 30-34.

18. Kolla S.B., Aggarwal S., Kumar A. et al. Early versus delayed laparoscopic cholecystectomy for acute cholecystitis: a prospective randomized trial // Surg. Endosc., 2004, 18, pp. 1323-1327.

19. Yadav R.P., Adhikary S., Agrawal C.S. et al. A comparative study of early vs. delayed laparoscopic cholecystectomy in acute cholecystitis // Kathmandu Univ. Med. J. (KUMJ), 2009, 7, p. 16-20.

20. Mare L.D., Saadi A., Roulin D. et al. Delayed versus early laparoscopic cholecystectomy for acute cholecystitis: a prospective randomized study // HPB, 2012, 14(Suppl), p. 130.

21. Gul R., Dar R.A., Sheikh R.A et al. Comparison of early and delayed laparoscopic cholecystectomy for acute cholecystitis: experience from a single center // N. Am. J. Med. Sci., 2013, 5, pp. 414-418.

22. Gutt C.N., Encke J., Köninger J. et al. Acute cholecystitis: early versus delayed cholecystectomy, a multicenter randomized trial (ACDC study, NCT00447304) // Ann. Surg., 2013, 258, pp. 385-393.

23. Agrawal R., Sood K.C., Agarwal B. Evaluation of early versus delayed laparoscopic cholecystectomy in acute cholecystitis // Surg. Res. Pract., 2015, 2015:349801.

24. Saber A., Hokkam E.N. Operative outcome and patient satisfaction in early and delayed laparoscopic cholecystectomy for acute cholecystitis // Minim. Invasive Surg., 2014, 2014: 162643.

25. Rajcok M., Bak V., Danihel L., et al. Early versus delayed laparoscopic cholecystectomy in treatment of acute cholecystitis // Bratisl. Lek. Listy., 2016, 117(6), pp. 328-31. 
26. Roulin D., Saadi A., Di Mare L. et al. Early Versus Delayed Cholecystectomy for Acute Cholecystitis, Are the 72 hours Still the Rule?: A Randomized Trial // Ann. Surg., 2016, 264(5), pp. 717-722.

27. Sutton A.J., Vohra R.S., Hollyman M. et al. Cost-effectiveness of emergency versus delayed laparoscopic cholecystectomy for acute gallbladder pathology. // Br. J. Surg., 2016, 104(1), pp. 98-107.

\section{References}

1. Everhart J.E., Ruhl C.E. Burden of digestive diseases in the United States. Part III: liver, biliary tract and pancreas. Gastroenterology, 2009, 136, pp. 1134-1144.

2. Aerts R., Penninckx F. The burden of gallstone disease in Europe. Aliment. Pharmacol. Ther., 2003, 18 (Suppl. 3), pp. 49-53.

3. Vorotyntsev A. S. Sovremennye predstavleniya o diagnostike i lechenii zhelchnokamennoj bolezni i khronicheskogo kal'kuleznogo kholetsistita [Current ideas on the diagnosis and treatment of gallstone disease and chronic calculous cholecystitis]. Lechashhij vrach. 2012. №2, pp. 54-58.

4. Indar A.A., Beckingham I.J. Acute cholecystitis. BMJ: British Medical Journal, 2002, 325 (7365), pp. 639-643.

5. Beburishvili A.G., Prudkov M.I., Sovtsov S.A. et al. Natsional'nye klinicheskie rekomendatsii «Ostryj kholetsistit» [National guidelines "Acute cholecystitis"]. Trudy XII S" ezda khirurgov Rossii «Aktual'nye voprosy khirurgii» [Proc. 12th Congress of Russian surgeons "Actual question of surgery]. Rostov-na-Donu, 2015.

6. Yamashita Y., Takada T., Hirata K. A survey of the timing and approach to the surgical management of patients with acute cholecystitis in Japanese hospitals. $J$. Hepatobiliary Pancreat. Surg., 2006, 13, pp. 409-415.

7. Johansson M., Thune A., Blomqvist A., Nelvin L., Lundell L. Management of acute cholecystitis in the laparoscopic era: results of a prospective, randomized clinical trial. J. Gastrointest. Surg., 2003, 7, pp. 642-645.

8. Johansson M., Thune A., Blomqvist A, Nelvin L., Lundell L. Impact of choice of therapeutic strategy for acute cholecystitis on patient's health-related quality of life. Results of a randomized, controlled clinical trial. Dig. Surg., 2004, №21, pp. 359-362.

9. Macafee D.A., Humes D.J., Bouliotis G., Beckingham I.J., Whynes D.K., Lobo D.N. Prospective randomized trial using cost-utility analysis of early versus delayed laparoscopic cholecystectomy for acute gallbladder disease. Br. J. Surg., 2009, 96, pp. 1031-1040.

10. Ozkardes A.B., Tokaç M., Dumlu E.G., Bozkurt B., Ciftçi A.B., Yeti, sir F. et al. Early versus delayed laparoscopic cholecystectomy for acute cholecystitis: a prospective, randomized study. Int. Surg., 2014, 99, pp. 56-61. 
11. Zhou M.W., Gu X.D., Xiang J.B., Chen Z.Y. Comparison of clinical safety and outcomes of early versus delayed laparoscopic cholecystectomy for acute cholecystitis: a meta-analysis. ScientificWorldJournal, 2014, 2014:274516.

12. Menahem B., Mulliri A., Fohlen A. et al. Delayed laparoscopic cholecystectomy increases the total hospital stay compared to an early laparoscopic cholecystectomy after acute cholecystitis: an updated meta-analysis of randomized controlled trials. HPB (Oxford), 2015, 17(10), pp. 857-862.

13. Lai P.B., Kwong K.H., Leung K.L. et al. Randomized trial of early versus delayed laparoscopic cholecystectomy for acute cholecystitis. Br. J. Surg., 1998, 85, pp. 764-767.

14. Lo C.M., Liu C.L., Fan S.T. et al. Prospective randomized study of early versus delayed laparoscopic cholecystectomy for acute cholecystitis. Ann. Surg., 1998, 227, pp. 461-467.

15. Dávila D., Manzanares C., Picho M.L. et al. Experience in the treatment (early vs. delayed) of acute cholecystitis via laparoscopy. Cirugia Española, 1999, 66(Suppl 1), pp. 233.

16. Chandler C.F., Lane J.S., Ferguson P. et al. Prospective evaluation of early versus delayed laparoscopic cholecystectomy for treatment of acute cholecystitis. Am. Surg., 2000, 66, pp. 896-900.

17. Khan S.S.A. Early versus delayed cholecystectomy for acute cholecystitis, a prospective randomized study. Pak. J. Gastroenterol., 2002, 16, pp. 30-34.

18. Kolla S.B., Aggarwal S., Kumar A. et al. Early versus delayed laparoscopic cholecystectomy for acute cholecystitis: a prospective randomized trial. Surg. Endosc., 2004, 18, pp. 1323-1327.

19. Yadav R.P., Adhikary S., Agrawal C.S. et al. A comparative study of early vs. delayed laparoscopic cholecystectomy in acute cholecystitis. Kathmandu Univ. Med. J. (KUMJ), 2009, 7, pp. 16-20.

20. Mare L.D., Saadi A., Roulin D. et al. Delayed versus early laparoscopic cholecystectomy for acute cholecystitis: a prospective randomized study. $H P B, 2012$, 14(Suppl), p. 130.

21. Gul R., Dar R.A., Sheikh R.A et al. Comparison of early and delayed laparoscopic cholecystectomy for acute cholecystitis: experience from a single center. N. Am. J. Med. Sci., 2013, 5, pp. 414-418.

22. Gutt C.N., Encke J., Köninger J. et al. Acute cholecystitis: early versus delayed cholecystectomy, a multicenter randomized trial (ACDC study, NCT00447304). Ann. Surg., 2013, 258, pp. 385-393.

23. Agrawal R., Sood K.C., Agarwal B. Evaluation of early versus delayed laparoscopic cholecystectomy in acute cholecystitis. Surg. Res. Pract., 2015, 2015:349801. 
24. Saber A., Hokkam E.N. Operative outcome and patient satisfaction in early and delayed laparoscopic cholecystectomy for acute cholecystitis. Minim. Invasive Surg., 2014, 2014: 162643.

25. Rajcok M., Bak V., Danihel L., et al. Early versus delayed laparoscopic cholecystectomy in treatment of acute cholecystitis. Bratisl. Lek. Listy., 2016, 117(6), pp. 328-31.

26. Roulin D., Saadi A., Di Mare L. et al. Early Versus Delayed Cholecystectomy for Acute Cholecystitis, Are the 72 hours Still the Rule?: A Randomized Trial. Ann. Surg., 2016, 264(5), pp. 717-722.

27. Sutton A.J., Vohra R.S., Hollyman M. et al. Cost-effectiveness of emergency versus delayed laparoscopic cholecystectomy for acute gallbladder pathology. Br. J. Surg., 2016, 104(1), pp. 98-107.

\section{ДАННЫЕ ОБ АВТОРЕ}

Манзюк Алексей Васильевич, студент

ФГБОУ ВО Первый Московский Государственный Медицинский Университет им. И. М. Сеченова Минздрава России (Сеченовский Университет)

ул. Трубеикая, 8, г. Москва, 119991, Российская Федерация. aleksej-manzyuk@yandex.ru

\section{DATA ABOUT THE AUTHOR}

\section{Manziuk Aleksei Vasilevich, Student}

The first Moscow State Medical University after I.M. Sechenov, Ministry of health of the Russian Federation (Sechenovskiy University)

8, Trubetskaya str., Moscow, 119991, Russian Federation

aleksej-manzyuk@yandex.ru

SPIN-code: 9044-6712

ORCID: 0000-0003-4087-3351

ResearcherID: T-7949-2018 